OPEN ACCESS

Edited by:

Kathleen M. Gorman,

Temple Street Children's University

Hospital, Ireland

Reviewed by:

Aline L. Petrin,

The University of lowa, United States

Enrique Medina-Acosta,

State University of the North

Fluminense Darcy Ribeiro, Brazil

*Correspondence:

Jihong HaO

haojihong2003@163.com

tThese authors have contributed equally to this work and share first

authorship

Specialty section:

This article was submitted to

Genetics of Common and Rare

Diseases,

a section of the journal

Frontiers in Pediatrics

Received: 06 April 2021

Accepted: 15 June 2021

Published: 08 July 2021

Citation:

Hou X, Sun J, Liu C and Hao J (2021)

Case Report: Wiskott-Aldrich

Syndrome Caused by Extremely

Skewed X-Chromosome Inactivation

in a Chinese Girl.

Front. Pediatr. 9:691524.

doi: 10.3389/fped.2021.691524

\section{Case Report: Wiskott-Aldrich Syndrome Caused by Extremely Skewed X-Chromosome Inactivation in a Chinese Girl}

\author{
Xuening Hou ${ }^{1+}$, Jie Sun ${ }^{2+}$, Chen $\mathrm{Liu}^{1}$ and Jihong Hao ${ }^{1 *}$ \\ ${ }^{1}$ Department of Clinical Laboratory, The Second Hospital of Hebei Medical University, Shijiazhuang, China, ${ }^{2}$ Department of \\ Gynecological Ultrasound, The Second Hospital of Hebei Medical University, Shijiazhuang, China
}

Wiskott-Aldrich syndrome (WAS) is a rare X-linked immunodeficiency disorder caused by abnormal expression of Wiskott-Aldrich syndrome protein due to WAS gene mutation, which is generally characterized by microthrombocytopenia, eczema, recurrent infections, and high risk of autoimmune complications and hematological malignancies. Although affected males with WAS usually manifest severe symptoms, female carriers have no significant clinical manifestations. Here, we describe a Chinese girl diagnosed with WAS carrying a heterozygous missense mutation in exon 2 of the WAS gene. The patient presented with persistent thrombocytopenia with small platelets and decreased WAS protein detected by flow cytometry and western blot analysis. The methylation analysis of the HUMARA gene displayed an extremely skewed X-chromosome inactivation (SXCl) pattern, where the X-chromosomes bearing normal WAS gene were predominantly inactivated, leaving the mutant gene active. Hence, our results suggest that completely inactivating the unaffected paternal X-chromosomes may be the reason for such phenotype in this female patient. SXCI has important implications for genetic counseling of female carriers with a family history of WAS.

Keywords: Wiskott-Aldrich syndrome, microthrombocytopenia, female carrier, heterozygous mutation, $\mathrm{X}$-chromosome inactivation

\section{INTRODUCTION}

Wiskott-Aldrich syndrome (WAS, OMIM 301000) is a rare X-linked recessive immunodeficiency disorder characterized by thrombocytopenia with small platelets, eczema, and recurrent infections. The clinical phenotype has a wide spectrum, varying from mild thrombocytopenia to severe clinical manifestations, including life-threatening hemorrhages, immunodeficiency, atopy, autoimmunity, and malignancies (1). The WAS gene, whose mutations are associated with the clinical phenotype of WAS, is mapped to Xp11.23, and encodes for the Wiskott-Aldrich syndrome protein (WASp). The severity of WAS phenotype in patients correlates with specific mutations and the expression levels of WASp in the blood cells. Usually, affected males show symptoms at an early age, while females carrying the defective WAS gene are asymptomatic. Till date, fewer than 20 female cases have been reported with WAS worldwide (Supplemental Table 1). Most studies favor the hypothesis that the clinical phenotype of female carriers is attributed to skewed X-chromosome inactivation (XCI), 
where the mutated $\mathrm{X}$-chromosomes are preferentially selected to be active (2-6).

Herein, we describe a 9-year-old Chinese girl with WAS who presented with congenital thrombocytopenia and hemorrhagic events. The patient had a heterozygous mutation of the WAS gene on the maternally derived X-chromosome, associated with the paternal X-chromosome completely inactive in the peripheral blood cells.

\section{CASE REPORT}

In 2019, a 9-year-old girl was admitted to the hospital because
A

\section{I}

II

III

IV

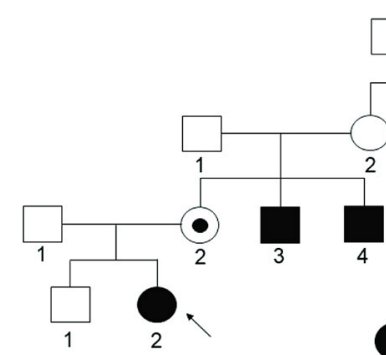

C

Control

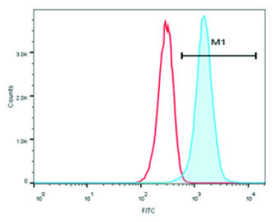

Mother

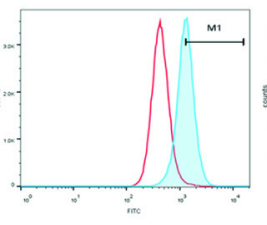

proband

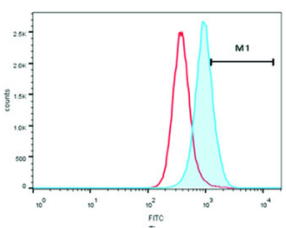

B
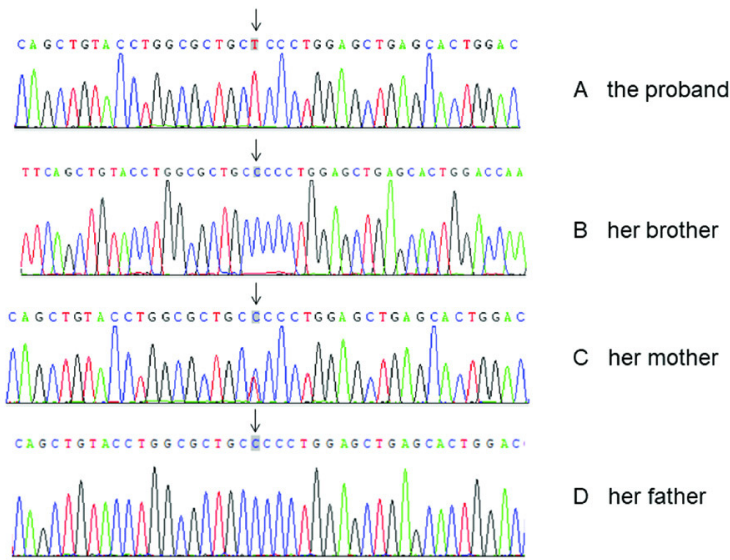

B her brother

$C$ her mother

D her father
D

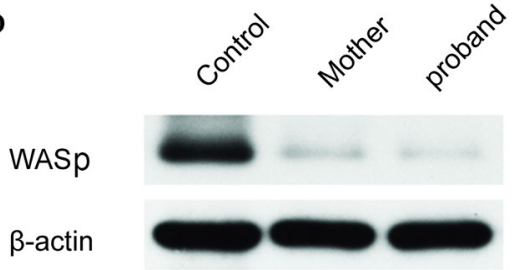

E
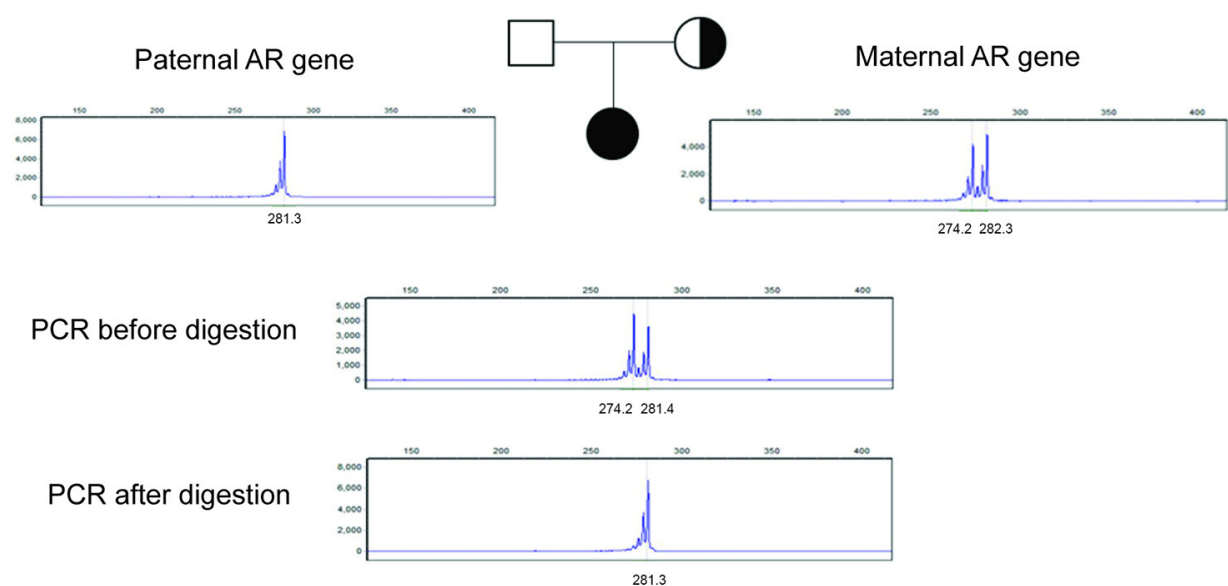

FIGURE 1 | (A) The pedigree of the patient's family. III.2: female carrier; III.3, III.4, and III.5: affected male; IV.2: female proband. (B) Sanger sequencing of the WAS gene of C.173C>T. The proband and her mother were heterozygous of the mutated gene, and her older brother and her father were normal. (C) WASp expression levels in the PBMCs by flow cytometry assay. The proportion of M1 area was $98.5 \%$ of normal control, $50.6 \%$ of her mother and $20.6 \%$ of the proband, respectively. (D) WASp expression levels were analyzed by western blotting. The gray mean value of the proband's mother is $10.8 \%$ of the control level and the gray mean value of proband is $5.4 \%$ of the level. (E) X-chromosome inactivation analysis of the patient and her parents. The experiments were performed in triplicate. 
TABLE 1 | Immunologic and hematologic characteristic of the patient and her mother.

\begin{tabular}{lccc}
\hline Variable & Patient & The patient's mother & Normal range \\
\hline Platelets & & & \\
Count $\left(\times 10^{9} / \mathrm{L}\right)$ & 19 & 9.1 & $125-350$ \\
Mean Volume (fL) & 5.9 & & $7.4-11.0$ \\
Serum immunoglobins (g/L) & & 11.97 & \\
IgG & 3.78 & 0.52 & $7.5-15.0$ \\
IgM & 0.14 & 0.34 & $1.0-5.0$ \\
IgA & 1.74 & & $0.46-3.0$ \\
Lymphocyte phenotype (\%) & & 66.9 & $20-50$ \\
Total T cells & 77.9 & 38.5 & $33-58$ \\
CD3+CD4+ & 48.7 & 21.7 & $20-39$ \\
CD3+CD8+ & 26.9 & 1.77 & $0.7-2.5$ \\
CD3+CD4+/CD3+CD8+ & 1.81 & 7.6 & $5-18$ \\
CD19+/CD20+ & 9.9 & 23.7 & $10-20$ \\
CD3-CD56+ & 11.4 & & \\
\hline
\end{tabular}

of severe colporrhagia for 3 days. She was initially admitted to the hospital due to thrombocytopenia at the age of 4 months and was diagnosed with immune thrombocytopenia (ITP). However, she failed to recover with hormone and human immunoglobulin injections, and her platelet count fluctuated between $20 \times$ $10^{9} / \mathrm{L}$ and $30 \times 10^{9} / \mathrm{L}$. She then experienced frequent fever, followed by recurrent administration of anti-infective drugs due to upper respiratory tract infection. One and a half months ago, she was admitted to a local hospital for respiratory infection before being discharged with improved condition through antiinfection treatment.

Physical examination revealed petechiae on both eyelids and upper limbs, bulbous conjunctival hemorrhage and pharyngeal hyperemia, and tonsil $\mathrm{I}^{\circ}$ swelling, although no eczema was noted. Peripheral blood cell count demonstrated $16.91 \times 10^{9} / \mathrm{L}(3.5-$ $\left.9.5 \times 10^{9} / \mathrm{L}\right) \mathrm{WBC}, 139 \mathrm{~g} / \mathrm{L}(120-140 \mathrm{~g} / \mathrm{L})$ hemoglobin, and 19 $\times 10^{9} / \mathrm{L}\left(125-350 \times 10^{9} / \mathrm{L}\right)$ platelet with the low mean platelet volume (MPV) of $5.90 \mathrm{fL}$ (7.4-11.0 fL). Small platelets were found in the peripheral blood smears.

Her serum IgG and IgM levels were lower than normal (3.78 and $0.14 \mathrm{~g} / \mathrm{L}$, respectively). Mycoplasma pneumoniae antibodies were positive, with the titer of 1:160, and without platelet-associated antibodies. Analysis of the lymphocyte subsets showed elevated total $\mathrm{T}$ cell count without any immunologic abnormalities. Bone marrow aspirate exhibited delayed maturation of megakaryocytes. Combined with her medical history and ineffective hormonal treatment, she was suspected of having inherited platelet disorders.

Upon investigating her family history, her father and elder brother showed no symptoms (Figure 1A), while her mother showed decreased IgM and IgA levels, with normal PLT. The detailed results are shown in Table 1. Her three maternal uncles presented thrombocytopenia for many years.

Sanger sequencing revealed a heterozygous missense mutation in exon 2 of the WAS gene, leading to a change from proline to leucine at position 58 in the patient and her mother (Figure 1B).

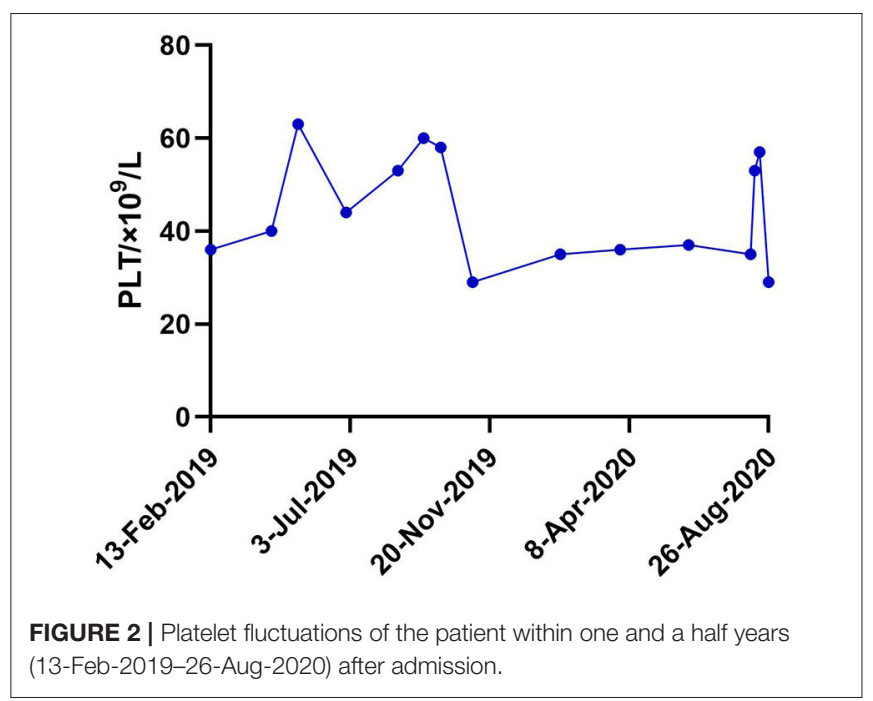

No mutations were detected in her father or elder brother. Meanwhile, flow cytometry showed the level of WASp from the proband and in the cells from her mother at 20.6 and $50.4 \%$, respectively (Figure 1C). The ratio of WASp was reduced by $\sim 5$-fold in mononuclear cells from the proband compared to the normal control (98.5\%). Western blot analysis revealed significantly decreased WASp levels in the patient and her mother compared to normal control (Figure 1D). Based on the above findings, the girl was confirmed to have WAS. Her platelet count fluctuated between $30 \times 10^{9} / \mathrm{L}$ and $60 \times 10^{9} / \mathrm{L}$ within one and a half years after admission (Figure 2). At present, the patient is receiving symptomatic treatment and awaiting hematopoietic stem cell transplantation.

Moreover, XCI analysis was performed using the human androgen receptor assay (HUMARA). Briefly, genomic DNA was extracted from peripheral blood leukocytes and used to amplify the highly polymorphic CAG repeat in the first exon of the HUMARA gene on Xq11-q12 (4). The degree of X-inactivation was calculated from the peak height. The results showed that the proband had only one paternal allele amplified after HpaII digestion (Figure 1E). In contrast, her mother showed two bands in the presence of HpaII; however, the rate of random inactivation indicated a mildly skewed pattern of XCI in mature blood cells (Figure 1E). The rate of XCI was 100 and $62.7 \%$ for the patient and her mother, respectively (Table 2 ).

\section{DISCUSSION}

Herein, we present a Chinese girl with a congenital thrombocytopenia, hemorrhagic tendency, and recurrent infections with c.173C $>\mathrm{T}$, a missense mutation of the WAS gene. Although missense mutations are most common in patients with WAS, this mutation is so rare that only three cases have been reported in the USA, China, and Brazil (7-9). Unlike the three male cases, our patient was a girl with this mutation. The c. $173 \mathrm{C}>\mathrm{T}$ mutation reported here is in the second exon that falls in the WH1 domain, which binds to the site of WASp interacting 
TABLE 2 | The results of X-chromosome inactivation of the patient and her mother.

\begin{tabular}{|c|c|c|c|c|c|c|c|c|}
\hline & $\begin{array}{c}\text { PCR } \\
\text { fragment } \\
\text { location (-) }\end{array}$ & $\begin{array}{l}\text { PCR fragment } \\
\text { location }(+)\end{array}$ & $\begin{array}{c}\text { Peak } 1 \\
\text { height }(+)\end{array}$ & $\begin{array}{c}\text { Peak } 2 \\
\text { height }(+)\end{array}$ & $\begin{array}{c}\text { Peak } 1 \\
\text { height (-) }\end{array}$ & $\begin{array}{c}\text { Peak } 2 \\
\text { height (-) }\end{array}$ & $\begin{array}{l}\text { Rate of random } \\
\text { inactivation }\end{array}$ & $\begin{array}{c}\text { The proportional } \\
\text { relation of } \\
\text { random } \\
\text { inactivation }\end{array}$ \\
\hline \multirow[t]{2}{*}{ IV2 } & n1 274.2 & / & 0 & 1,301 & 580 & 770 & $100 \%$ & 100:0 \\
\hline & n2 281.4 & 281.3 & & & & & & \\
\hline \multirow[t]{2}{*}{ III2 } & n1 274.2 & 274.1 & 969 & 824 & 1,173 & 1,686 & $62.8 \%$ & 63:37 \\
\hline & n2 282.3 & 282.1 & & & & & & \\
\hline III1 & n1 281.3 & & & & & & & \\
\hline
\end{tabular}

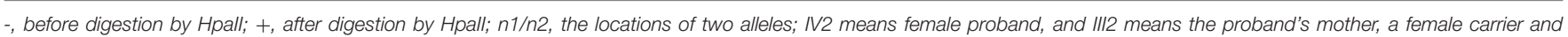

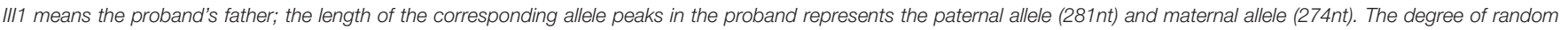

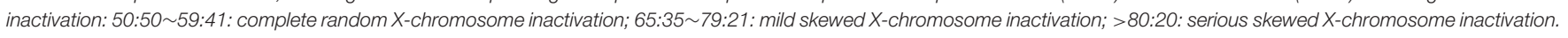

protein (WIP), crucially influencing the stability of WASp. Moreover, this domain plays a critical role in a series of events, including intracellular signal transduction, protein folding, and transport (10).

The WAS gene located on the $\mathrm{X}$-chromosome contributed to WAS, which is transmitted in compliance with an X-linked recessive inheritance feature. X-linked female carriers show no clinical manifestations due to the pattern of XCI, which may ameliorate the destructive effects of the X-linked mutations (11). Nevertheless, XCI maintenance mechanisms are remarkably diverse, which closely affects $\mathrm{X}$-linked gene dosage and sex differences in X-linked diseases (12). Therefore, the pattern of $\mathrm{XCI}$ may aggravate the clinical manifestations of the disease. Our present study proposed that skewed XCI led to the clinical phenotype of this female patient with WAS. In some cases, this is possibly due to genetic defects in the $\mathrm{X}$-inactivation transcriptional process, contributing to the unusually skewed XCI pattern (4). The imbalanced expression of the parental Xchromosomes seems to be common in the general population; however, the skewed XCI pattern increased the risk of recessive $\mathrm{X}$-linked disorders in females (13).

The results of XCI analysis showed two peaks at different positions in the proband and her mother before digestion with HpaII, indicating that the alleles were derived from both paternal and maternal chromosomes. However, the proband had only one allele left after enzymatic digestion, demonstrating that the proband had preferential inactivation of the paternally derived wild-type allele. The selection of $\mathrm{XCI}$ was independent of the mutation originating from either parent. While the spontaneous heterozygous mutation of the WAS gene was on the paternally derived X-chromosome, the extremely skewed XCI preferentially selected the maternally derived wild-type $\mathrm{X}$-chromosome to be inactivated (5). From a comprehensive perspective, the genetic basis of the clinical presentation of WAS in our proband is the fortuitous combined occurrence of a maternally inherited mutation and the extreme skewing of inactivation against the unaffected paternal chromosome.

The proportion of inactivation was $100 \%$ in the patient and $62.7 \%$ in her mother (Table 2), manifesting the overwhelmingly skewed XCI of the patient and slight inactivation of her mother.
These results were consistent with the expression levels of WASp. At the cytogenetic level, it also explained the differences in clinical signs between the mother and daughter, who were both carriers of heterozygous mutation.

Based on WASp-deficient mice, the skewed inactivation of WAS carriers was attributed to homing deficits in fetal hematopoietic stem cells (14). In addition, the selected inactivation can be present at a later stage, even in mature populations of hematopoietic differentiation (15). Thus, the predominant selection against mutated alleles as well as skewed inactivation can occur throughout the progress of hematopoietic development, increasing the risk of WAS in females. Diverse patterns of XCI exist in female carriers, such as random, slightly skewed, or extremely skewed patterns $(3,16)$. As a heterozygous carrier, the patient's mother exhibited slightly skewed inactivation in our study. This illustrates that the XCI pattern in humans shows diversity between individuals without stochastic mechanisms. In addition, a previous study indicated that the blood cell population bearing the mutated WAS message was greater in patients with WAS than in their mothers (3). Hence, the degree of imbalanced XCI in WAS carriers may vary with age. A WAS carrier has been reported to present random XCI in granulocytes and non-random inactivation in $\mathrm{T}$ lymphocytes (15). In contrast, a female with X-linked thrombocytopenia was also reported with random inactivation in lymphocytes along with skewed XCI in buccal mucosal cells (17). Therefore, the XCI pattern also varies at the cellular level and across different cell types.

In summary, the cumulative clinical results and molecular assays illustrated that a female carrier with a heterozygous WAS mutation could express an X-linked WAS disease. To the best of our knowledge, the case reported here is the first Chinese female patient presenting WAS clinical symptoms with an extremely skewed XCI pattern, in which the mutated allele was preferentially active. XCI analysis is a relatively effective and rapid technique to analyze the pattern of XCI in female carriers, who are suspected of having an X-linked recessive disorder. Furthermore, this case reminds us of the fact that female carriers with a family history of WAS may also develop certain clinical symptoms. 


\section{DATA AVAILABILITY STATEMENT}

The original contributions presented in the study are included in the article/Supplementary Material, further inquiries can be directed to the corresponding author/s.

\section{ETHICS STATEMENT}

The studies involving human participants were reviewed and approved by the Research Ethics Committee of the Second Hospital of Hebei Medical University. Written informed consent to participate in this study was provided by the participants' legal guardian/next of kin. Written informed consent was obtained from the individual(s), and minor(s)' legal guardian/next of kin, for the publication of any potentially identifiable images or data included in this article.

\section{AUTHOR CONTRIBUTIONS}

All the authors have accepted responsibility for the entire content of this submitted manuscript and approved submission. $\mathrm{XH}$

\section{REFERENCES}

1. Candotti F. Clinical manifestations and pathophysiological mechanisms of the Wiskott-Aldrich syndrome. J Clin Immunol. (2018) 38:13-27. doi: 10.1007/s10875-017-0453-z

2. Parolini O, Ressmann G, Haas OA, Pawlowsky J, Gadner H, Knapp W, et al. X-linked Wiskott-Aldrich syndrome in a girl. N Engl J Med. (1998) 338:291-5. doi: 10.1056/NEJM199801293380504

3. Ariga T, Yamada M, Wada T, Saitoh S, Sakiyama Y. Detection of lymphocytes and granulocytes expressing the mutant WASP message in carriers of Wiskott-Aldrich syndrome. Br J Haematol. (1999) 104:893900. doi: 10.1046/j.1365-2141.1999.01248.x

4. Andreu N, Pujol-Moix N, Martinez-Lostao L, Oset M, Muñiz-Diaz E, Estivill X, et al. Wiskott-Aldrich syndrome in a female with skewed X-chromosome inactivation. Blood Cells Mol Dis. (2003) 31:332-7. doi: 10.1016/s1079-9796(03)00168-2

5. Boonyawat B, Dhanraj S, Al Abbas F, Zlateska B, Grunenbaum E, Roifman CM, et al. Combined de-novo mutation and non-random $\mathrm{X}$-chromosome inactivation causing Wiskott-Aldrich syndrome in a female with thrombocytopenia. J Clin Immunol. (2013) 33:1150-5. doi: 10.1007/s10875-013-9927-9

6. Takimoto T, Takada H, Ishimura M, Kirino M, Hata K, Ohara O, et al. Wiskott-Aldrich syndrome in a girl caused by heterozygous WASP mutation and extremely skewed X-chromosome inactivation: a novel association with maternal uniparental isodisomy 6. Neonatology. (2015) 107:18590. doi: 10.1159/000370059

7. Kwan SP, Hagemann TL, Radtke BE, Blaese RM, Rosen FS. Identification of mutations in the Wiskott-Aldrich syndrome gene and characterization of a polymorphic dinucleotide repeat at DXS6940, adjacent to the disease gene. Proc Natl Acad Sci USA. (1995) 92:4706-10. doi: 10.1073/pnas.92.10.4706

8. Zhang J, Shi J, Li X, Shao Y, Liu C, Ge M, et al. The gene mutation analysis of a Wiskott-Aldrich syndrome family with normal mean platelet volume. Zhonghua Xue Ye Xue Za Zhi. (2015) 36:7548. doi: 10.3760/cma.j.issn.0253-2727.2015.09.007

9. Medina SS, Siqueira LH, Colella MP, Yamaguti-Hayakawa GG, Duarte BKL, Dos Santos Vilela MM, et al. Intermittent low platelet counts hampering diagnosis of X-linked thrombocytopenia in children: report of two unrelated cases and a novel mutation in the gene coding for the Wiskott-Aldrich syndrome protein. BMC Pediatr. (2017) 17:151. doi: 10.1186/s12887-017-0897-6 drafted the manuscript. JS and CL contributed to the design, acquisition, and analysis of data. JH final approval of the version to be published and offered professional guidance.

\section{FUNDING}

This study was supported by grants from the Natural Science Foundation of Hebei Province of China (H2020206349) and the Research Fund of the Second Hospital of Hebei Medical University of China (2h2019041).

\section{ACKNOWLEDGMENTS}

We would like to thank all members of the study team, the patient and her family.

\section{SUPPLEMENTARY MATERIAL}

The Supplementary Material for this article can be found online at: https://www.frontiersin.org/articles/10.3389/fped. 2021.691524/full\#supplementary-material

10. Matalon O, Reicher B, Barda-Saad M. Wiskott-Aldrich syndrome proteindynamic regulation of actin homeostasis: from activation through function and signal termination in T lymphocytes. Immunol Rev. (2013) 256:1029. doi: $10.1111 /$ imr. 12112

11. Migeon BR. The role of $\mathrm{X}$ inactivation and cellular mosaicism in women's health and sex-specific diseases. JAMA. (2006) 295:1428-33. doi: 10.1001/jama.295.12.1428

12. Carrel L, Willard HF. X-inactivation profile reveals extensive variability in $\mathrm{X}$-linked gene expression in females. Nature. (2005) 434:400-4. doi: 10.1038/nature03479

13. Shvetsova E, Sofronova A, Monajemi R, Gagalova K, Draisma HHM, White SJ, et al. Skewed X-inactivation is common in the general female population. Eur J Hum Genet. (2019) 27:455-65. doi: 10.1038/s41431-018-0291-3

14. Lacout C, Haddad E, Sabri S, Svinarchouk F, Garçon L, Capron C, et al. A defect in hematopoietic stem cell migration explains the nonrandom $\mathrm{X}$ chromosome inactivation in carriers of Wiskott-Aldrich syndrome. Blood. (2003) 102:1282-9. doi: 10.1182/blood-2002-07-2099

15. Notarangelo LD, Parolini O, Porta F, Locatelli F, Lanfranchi A, Marconi M, et al. Analysis of X-chromosome inactivation and presumptive expression of the Wiskott-Aldrich syndrome (WAS) gene in hematopoietic cell lineages of a thrombocytopenic carrier female of WAS. Hum Genet. (1991) 88:23741. doi: 10.1007/BF00206081

16. de Saint Basile G, Lagelouse RD, Lambert N, Schwarz K, Le Mareck B, Odent $\mathrm{S}$, et al. Isolated X-linked thrombocytopenia in two unrelated families is associated with point mutations in the Wiskott-Aldrich syndrome protein gene. J Pediatr. (1996) 129:56-62. doi: 10.1016/s0022-3476(96)70190-7

17. Inoue $H$, Kurosawa $H$, Nonoyama S, Imai K, Kumazaki H, Matsunaga $T$, et al. X-linked thrombocytopenia in a girl. Br J Haematol. (2002) 118:11635. doi: 10.1046/j.1365-2141.2002.03740.x

Conflict of Interest: The authors declare that the research was conducted in the absence of any commercial or financial relationships that could be construed as a potential conflict of interest.

Copyright (C) 2021 Hou, Sun, Liu and Hao. This is an open-access article distributed under the terms of the Creative Commons Attribution License (CC BY). The use, distribution or reproduction in other forums is permitted, provided the original author(s) and the copyright owner(s) are credited and that the original publication in this journal is cited, in accordance with accepted academic practice. No use, distribution or reproduction is permitted which does not comply with these terms. 